\title{
Paracelsus im Urteil des 18. Jahrhunderts
}

Dietrich von Engelhardt

\section{SUMMARY}

Paracelsus was a topic of intense discussion during the eighteenth century in the encyclopedias, works of philosophy, natural science and medicine of this time. His contributions to chemistry and the chemical foundation of medicine were acknowledged as well as his fight against the ancient humoral pathology and specific achievements in surgery and drug therapy. In contrast, his concept of science and use of language were rejected. The gap between his expressed moral standards and his own behavior was criticized. The contemporary circumstances must be taken into consideration.

\section{ZUSAMMENFASSUNG}

Paracelsus wird im 18. Jahrhundert in Enzyklopädien, Werken der Philosophie, Naturwissenschaften und Medizin diskutiert. Anerkennung können die Beiträge zur Chemie und chemischen Grundlegung der Medizin, zur Überwindung der antiken Humoralpathologie sowie spezifische Verdienste in der Chirurgie und medikamentösen Therapie finden, auf Ablehnung stossen sein Wissenschaftsbegriff, Vernunftverständnis und Sprachgebrauch, bedauert wird das Auseinanderfallen von ethischen Forderungen und eigenem Verhalten und verlangt die Beachtung der Zeitverhältnisse.

\section{Spektrum und Hintergrund}

Epochen, Schulen, Fächer und Individuen besitzen unterschiedliche und für sie jeweils charakteristische Urteile über Paracelsus. Zugleich fallen Darstellung und Deutung oft vielfältiger aus, als diese Voraussetzungen und Bindungen schliessen lassen. Paracelsus im Urteil der Aufklärung verlangt ebenso noch nach Differenzierung vorherrschender Auffassung wie Paracelsus im Urteil der Romantik und des Idealismus oder des positivistischen 19. Jahrhunderts ${ }^{1}$. 
Der Charakter dieses Beitrages kann nur paradigmatisch sein. Quellenforschung muss vor allem noch auf der Ebene der Akademie- und Gesellschaftsschriften sowie im Bereich der Zeitschriften geleistet werden; zahlreiche Monographien aus den verschiedenen Fachrichtungen müssen aber ebenfalls noch untersucht werden. Der Blick muss auch noch, wofür hier nur wenige Beispiele gegeben werden, über Deutschland, Frankreich und England hinaus auf andere Länder in Süd-, Ost- und Nordeuropa gerichtet werden. Leitend sind für die folgende Darstellung die allgemeinen Gesichtspunkte: Leben und Persönlichkeit, Werk und Wirkung, vor allem im Bereich der Naturforschung und Medizin.

Mit wiederholten Auflagen wird im übrigen die Jahrhundertgrenze 1700 überbrückt oder relativiert. Moreris «Grand Dictionaire historique` mit einem knappen Eintrag zu Paracelsus erscheint von 1674 bis 1732 in 18 Auflagen; Teissiers «Les Eloges des hommes savans» in der Nachfolge von de Thou (Historiae sui temporis, 1604-14) wird nach 1683 in vierter Auflage 1715 gedruckt; die Darstellung von Paracelsus gerät im allgemeinen positiv («savoir extraordinaire», «cures merveilleuses», «grande réputation»), negative Züge werden indessen nicht ausgespart («magicien», «presque yvre», «ereurs dangéreuses en matière de Religion» ${ }^{2}$ ). Conrings (Introductio in universam artem medicams erlebt nach 1654 und 1687 erneut 1726 eine Auflage, in der die abwertende Einschätzung von Paracelsus erhalten bleibt: «monstrum hominis \& in perniciem omnis melioris eruditionis natum» ${ }^{3}$. Morhof rechnet Paracelsus in seinem (Polyhistor) (1688/92, $\left.{ }^{4} 1747\right)$ zu den mystischen, theosophischen und magischen Schriftstellern, deren Werke ihre Verteidiger wie Kritiker gefunden hätten: «Varia de mysticis libris judicia. Non omnia contemnenda» ${ }^{4}$.Lionardo di Capoas «Parere, divisato in otto ragionamentis erscheint in erster Auflage 1681, in zweiter 1689 und in dritter 1695 (erneut 1714); Paracelsus, auf den mehrfach hingewiesen wird, habe die seit langem vernachlässigte Art des Philosophierens neu belebt und zur Lektüre des umfangreichen Buches der Natur angeregt («a legger il voluminoso libro della natura» ${ }^{5}$ ).

\section{Allgemeine Rezeption und Resonanz}

In den Enzyklopädien und Gelehrtenbiographien der Aufklärung, in den Werken der Philosophie und Geschichtswissenschaft dieses Jahrhunderts wird Paracelsus keineswegs übergangen oder nur negativ beurteilt. Unter- 
schiedliche Auffassungen kommen vor, insbesondere wird nach verschiedenen Dimensionen des Lebens und der Persönlichkeit, des Werkes und der Wirkung differenziert. In der «Cyclopaedia〉 (1728) von Chambers wird über Paracelsus, der auch im Artikel (Alchymy) genannt wird, ausgeführt, er habe sich mit van Helmont um die Chemie sehr verdient gemacht und "carry'd it to such a length, as to render medicine almost wholly chymica» ${ }^{6}$. In Zedlers 〈Universal-Lexikon〉 (1740) findet Paracelsus eine relativ neutrale Erwähnung ${ }^{7}$; er gilt als Haupt einer besonderen Sekte, seine Erfahrungen, die er aus der Natur gewonnen habe, werden ebenso hervorgehoben wie sein Konzept der Medizin mit den vier Säulen und seine therapeutischen Erfolge: «So viel ist indessen ganz gewiss, dass er viele Krankheiten mit besonderm Glück gehoben, welche andere Aerzte, die den Gebrauch des Opii und Quecksilbers damals noch nicht recht verstanden, gar nicht zu heilen wusten» ${ }^{8}$. In Jöchers 〈Allgemeinem Gelehrten-Lexikon〉 (1751) werden zustimmend der Hang von Paracelsus zur Chemie und seine Bereitschaft erwähnt, sich «so wohl von alten Weibern, als von Medicis, Chirurgis und Chymisten» belehren zu lassen. «Physic, Astronomie und Alchymie, nebst der Frömmigkeit» seien nach ihm die vier Hauptsäulen der Medizin, die er insgesamt auf der Basis der Chemie habe reformieren wollen. «Die Lehre von den drey Principiis der Chymicorum» ${ }^{9}$ sei allerdings nicht von ihm erfunden worden, sondern gehe auf Valentinus zurück, den er selbst jedoch nicht erwähnt habe.

Die Einschätzung in Diderots und d'Alemberts «Encyclopédie» (1765) fällt kritisch, aber nicht vollständig ablehnend aus. Nach einigen Hinweisen zu Leben und Werk wird ironisch daran erinnert, dass Paracelsus, der sich stets als Magiker («magicien», «sorcier») ausgegeben und gerühmt habe, den Stein der Weisen («remède universel») gefunden zu haben, bereits im Alter von 48 Jahren gestorben sei. In der Fülle der Absurditäten seiner Schriften kämen allerdings hin und wieder auch gute Einsichten vor, die zum Progress der Medizin beigetragen hätten, zur Überwindung zum Beispiel der antiken Humoralpathologie ${ }^{10}$.

In Sudens «Der gelehrte Criticus» (1717) werden an Paracelsus ein «vortreffliches Ingenium», hervorragendes Gedächtnis und grosse therapeutische Fähigkeiten gerühmt; er wollte nicht nur die Medizin reformieren, sondern «bemühte sich auch eine neue Theologie einzuführen» ${ }^{11}$; im übrigen sei er von der Möglichkeit der extrakorporalen Zeugung überzeugt gewesen: «Er schrieb und behauptete: Es könne ein Mensch durch Kunst und Natur auch ausser Mutter-Leibe generiret werden, und solches sey der Spagyri- 
schen Kunst, wie auch der Natur, keineswegs zuwider» ${ }^{12}$. Schröckh zählt Paracelsus in seinen «Abbildungen und Lebensbeschreibungen berühmter Gelehrten» (1764) in die «Klasse derjenigen, von denen die Urtheile der Welt nicht bloss verschieden, sondern ganz widersprechend und in einem übertriebenen Grade einander entgegen gesetzt $\sin d{ }^{13}$.

Von Savérien wird Paracelsus in der «Histoire des Philosophes modernes〉 (1769) unter die «chimistes et cosmologistes» gerechnet. Er habe erstaunliche theoretische Kenntnisse wie praktische Fähigkeiten in der Chemie besessen, sei aber im Stile der Zeit zu sehr der Magie verfallen gewesen: «il vivoit dans un temps où la vérité ne passoit point sans le merveilleux» ${ }^{14}$. Man müsse Paracelsus grosse Verdienste auch in der Medizin zugestehen, was allerdings nur zu oft nicht anerkannt werde: «Rendons plus de justice à ce grand homme» ${ }^{15}$. Erneut bestätige sich an Paracelsus die alte Wahrheit, dass es kein Genie ohne die Beimischung von Verrücktheit gebe («nullum magnum ingenium sine mixtura dementiae» $\left.{ }^{16}\right)$.

Während Paracelsus im 17. Jahrhundert bei Leibniz, der sich eingehend mit ihm beschäftigt, als Begründer einer «philosophischen Chymie» (1671), die zugleich die zentrale Voraussetzung des «wahren methodum medendi» abgebe, eine positive Einschätzung findet ${ }^{17}$, wird er bei den Philosophen des 18. Jahrhunderts im allgemeinen reserviert oder ablehnend beurteilt; empirisches Wissen kann ihm zuerkannt werden, sein Wissenschaftsverständnis und Vernunftbegriff werden dagegen verworfen.

Nach Walch (1726) steht Paracelsus mit seiner Lehre im Widerspruch zu Grundprinzipien der Philosophie; unter anderem habe er die «hermetische Physic» mit den drei Prinzipien Salz, Schwefel und Quecksilber «aus dem Staube wieder hervor gesuchet, dem hernach viele andere gefolget sind» ${ }^{18}$. Die paracelsische Auffassung von den Elementargeistern wird neutral wiedergegeben, zugleich jedoch eingewandt, Paracelsus habe «die BergMänngen mit den Pygmäis vermischet, welche von jenen, so viel wir aus den historischen Nachrichten sehen können, unterschieden sind» ${ }^{19}$. Brucker (1743) lobt zwar die Kenntnisse von Paracelsus in der Chemie («artis chemicae peritissimus fuerit» ${ }^{20}$ ), verurteilt aber entschieden seinen theosophischen Vernunftbegriff wie seine Wissenschaftssprache: «omnia tanta confusione et obscuritate explicat, ut Lectori sponte subnascatur suspicio, Paracelsum hunc librum ebrium dictitasse, nec intellexisse, quae in chartam referri iusserit. Multa vero inepta, superstitione plena, et vetulis, quam philosophis digniora in eo tractatu offenduntur» ${ }^{21}$. 
Adelung weist Paracelsus in seiner «Geschichte der menschlichen Narrheits (1789) einen prominenten Platz zu. Le Clercs Wiedergabe in seiner 〈Histoire de la médecine〉 $\left({ }^{2} 1723\right)$, der Adelung im medizinischen Bereich selbst weitgehend folgt, erhält seine ausdrückliche Zustimmung, nicht dagegen Reimmanns Bild (1713), der «von dem Marktschreyer in einem sehr erhabenen und panegyrischen Tone» ${ }^{22}$ gesprochen habe; ebensowenig können Adelung die Schilderungen Teissiers (1683), Sudens (1707), Stolles (1731), Du Fresnoys (1742), Kestners (1748), Leus (Bd.14, 1758), Eloys (1755) und Savériens (1769) wie die Angaben im Zedlerschen UniversalLexikon (Bd.26, 1740) oder Jöcherschen 〈Gelehrten-Lexikon〉 (Bd.3, 1751) überzeugen. Brucker (1743) sei «beinahe der erste gewesen», der die Aufgabe unternommen habe, «das Leben dieses Menschen und dessen System mit der Fackel der Kritik zu beleuchten» ${ }^{23}$. Paracelsus habe «unstät und armselig» ${ }^{24}$ gelebt, seine Lehre sei unsinnig und widersprüchlich gewesen: «die Verworrenheit in seinen Begriffen und in seinem Vortrage ist so gross, dass man auch zu oft den gemeinsten Menschenverstand vermisset. Sein Styl ist so roh, plump und ungebildet, als sein Kopf und seine Sitten» ${ }^{25}$.

Adelung steht aber nicht für das Jahrhundert insgesamt. 1776 lädt Wieland im «Teutschen Merkur` zu einer neuen Beurteilung von Paracelsus ein: «Paracelsus verdient aus zwei Ursachen einen Platz unter den Worthies den 16ten Jahrhunderts: als ein Mann von ausserordentlichem Genie, und weil er in der Artzneykunst Epoche gemacht» ${ }^{26}$. Die Philosophen des Deutschen Idealismus - nicht allerdings Kant ${ }^{27}$ und Fichte - wie Naturforscher und Mediziner der Romantik sind dieser Einladung in kongenialer Weise gefolgt.

Vielfältig ist während des 18. Jahrhunderts die Rezeption von Paracelsus oder vor allem seiner Elementargeister, vermittelt über «Le Comte de Gabalis ou entretiens sur les sciences secrètes> $(1670, \mathrm{dt}$. 1782) von de Villars, auch in Dichtung und Literatur - bei Pope (‘The Rape of the Lock», 1712), Wieland («Don Sylvio〉, 1764), Ditter von Dittersdorf (Singspiel «Doktor und Apotheker〉, 1786), von Matthisson («Die Elementargeister> 1799) und Goethe («Faust〉, 1790). Herder äussert sich skeptisch in seinen 〈Kritischen Wäldern (1769) über die Tendenz «Gnomen und Sylphen, und Nymphen und Salamander, die ganze Schöpfung des Theophrastus Paracelsus, und Cornelius Agrippa, die personificirte ganze Naturkunde in die Stelle mythologischer Wesen» treten zu lassen; «Fabel, Dichtung, Handlungen, die bis zur Täuschung eindringen, sind das Wesen der Dichtkunst, und wie weit weniger kann hier die Naturlehre zutragen $?{ }^{28}$. 


\section{Aufnahme und Beurteilung in Naturforschung und Medizin}

In den medizinhistorischen Schriften des 18. Jahrhunderts, nicht immer von Medizinern verfasst, wird Paracelsus fast durchgängig behandelt, werden sein Leben und seine Persönlichkeit, sein Werk und seine Wirkung wiederholt beschrieben und beurteilt; der Bogen spannt sich von Barchusen (1710, 1713), Reimmann (1713) und Le Clerc ( $\left.{ }^{2} 1723\right)$ über Kestner (1740, 1748) und Eloy (1755) zu Black (1782), Hemmann (1778) und Sprengel (1794), um nur einige wichtige Namen zu nennen.

Paracelsus kann in medizinhistorischen Werken des 18. Jahrhunderts aber auch unerwähnt bleiben oder nur äusserst knapp erwähnt werden. Freind geht auf Paracelsus, der von ihm in den 〈Praelectiones Chymicae〉 (1726) genannt wird, in seinem repräsentativen Werk 'The history of physick> (1725/26) nicht ein. Albrecht von Haller setzt seinem Schriftenverzeichnis des Paracelsus in der <Bibliotheca Chirurgica〉 (1774-75) eine konzentrierte und keineswegs nur negative Charakteristik voraus: «Magnus peregrinator, chemicus et astrologus, mirus homo, qui antiquos errores studeret subruere, ut suos substitueret» ${ }^{29}$. Eine 〈Litterargeschichte des Theophrastus Paracelsus» wird von Murr 1799 vorgelegt : «Ich setze chronologisch das Leben des Mannes zur Uebersicht hieher, zeige sodann den Zustand der damaligen medicinischen Kenntnisse, und schliesse mit einem genauen Verzeichnisse seiner Schriften» ${ }^{30}$. Bei Ackermann («Institutiones historiae medicinae», 1792) heisst es lakonisch: «chemicorum sectae in Germania auctor» ${ }^{31}$. Heckers «Allgemeine Geschichte der Natur- und Arzneykundes von 1793 bleibt unvollendet, kommt über die griechische Zeit nicht hinaus. Nach der einleitenden Übersicht markiert Paracelsus für Hecker die fünfte Periode, die bereits für sich schon, vor allem aber im Blick auf die folgende sechste oder chemische Periode wichtig gewesen sei. Mehrfach wird mit Paracelsus eine Epochenzäsur verbunden.

Auch in den wissenschaftshistorischen, vor allem chemiehistorischen Beiträgen des 18. Jahrhunderts, bei Bergman (1782), Gmelin (1797) oder Wiegleb $(1777,1781)$ kommt es zu Beurteilungen von Paracelsus. Vielseitig ist schliesslich ebenfalls die Resonanz in naturwissenschaftlichen, pharmazeutischen, medizinischen und medizintheoretischen Schriften der Zeit. In den chemischen Artikeln der ‘Encyclopédie〉 von Diderot und d'Alembert wird Paracelsus mehrfach erwähnt. Wiederholt wird auf ihn auch in chemischen Zeitschriften des ausgehenden 18. Jahrhunderts eingegangen. 
Auf die anhaltende Resonanz in alchemischen Schriften sei an dieser Stelle nur hingewiesen. Die Trennlinie zur empirisch-wissenschaftlichen Chemie kann in den ersten Jahrzehnten des 18. Jahrhunderts bekanntlich nicht immer scharf gezogen werden, der Alchemie werden vielmehr weiterhin reale Verdienste zugesprochen. Wiedergabe und Einschätzung von Paracelsus bleiben davon nicht unberührt.

Die normativen Standpunkte, die den Urteilen über Paracelsus zugrundeliegen, weichen voneinander ab. Bedenklichkeiten in Leben und Persönlichkeit gesteht Reimmann Paracelsus ebenso zu wie Widersprüche in der Lehre: "Allein das waren die Unvollkommenheiten die sich bey seiner Vollkommenheit befunden. Er war in seinen Gedanken hoffärtig, in seinen Worten zu grosssprecherisch, in seinen Werken zu eigensinnig, in seinen Sitten zu Baurisch, in seinen Principiis zu paradoxisch ${ }^{32}$.

Le Clerc, wiederholt mit seiner Darstellung $\left({ }^{2} 1723\right)$ während des 18. Jahrhunderts aufgegriffen, referiert die Vorwürfe vergangener Schriftsteller, distanziert sich von einigen, hält andere für berechtigt: «Ce que j'ai raporté jusqu'ici de Paracelse ne lui est pas avantageux. Cependant il faut avouer qu'il s'étoit acquis de bonne heure une très-grande réputation par sa Médecine» ${ }^{33}$. Auf der Basis von Le Clerc gibt Boerhaave in seinen «Elementa Chemiaes (1732) die wesentlichen Stationen des Lebens von Paracelsus wieder; das vorzeitige Ende in Salzburg habe seinen eigenen Versprechungen («quum solo Elixire proprietatis annos sibi Methusalemi promisisset» ${ }^{34}$ ) allerdings nicht entsprochen.

Die moralischen Vorwürfe, die auf eine Fülle überlieferter Anekdoten zurückgehen, werden im 18. Jahrhundert wiederholt, aber ebenso auch relativiert oder zurückgewiesen. Kestner möchte die Frage, ob Paracelsus «den Stein der Weisen besessen, mit dem Teufel ein Bündnis gehabt, und ein Castrat gewesen», andere untersuchen lassen. Nach Eloy - seinerseits in wörtlicher Nähe zu Le Clerc - hat Paracelsus eine «imagination, vive, mais déréglée, et la tête pleine d'idées creuses et chimériques» ${ }^{35}$ besessen. Venel (1753) urteilt komplex : «Paracelse est un des plus singuliers personnages que nous présente l'histoire littéraire: visionnaire, superstitueux, entêté des chimères de l'Astrologie, de la cabale, de la magie, de toutes les sciences occultes; mais hardi, présomptueux, enthousiaste, fanatique, extraordinaire en tout» ${ }^{36}$. Vernichtend äussert sich Zimmermann (1763): «Übrigens lebte er wie ein Schwein, sah aus wie ein Fuhrmann, und fand sein grösstes Vergnügen in dem Umgang des liederlichsten und niedrigsten Pöbels» ${ }^{37}$. Nach Carbonarius (1785) hat Paracelsus «einen geheimen und vertrauten 
Umgang mit dem Teufel, nicht nur für erlaubt, sondern auch unter gewissen Umständen für nothwendig» ${ }^{38}$ gehalten, er habe, wobei vor allem Oporins Bericht (26. 11. 1555) herangezogen wird, einen «lieblosen und menschenfeindlichen Charakter» ${ }^{39}$ besessen und sei «bey seiner groben Unwissenheit der unverschämteste Prahler, den jemals die Erde erzeugt hat» ${ }^{40}$, gewesen. Die Verwirklichung der vierten Säule der Medizin (Tugend) sei ihm misslungen, «da sein schändlicher Wandel die vierte Grundstütze untergrub, aushob und gänzlich zertrümmerte» ${ }^{41}$. Blumenbach (1786) sieht in Paracelsus einen «homo quidem paradoxus, indoctus» ${ }^{42}$, einen philosophischen Schwärmer, "vagabundus, combibo, moribus et stylo rusticus», zugleich aber «magni certe ingenii vir» ${ }^{43}$.

Mit neuen Strömungen wie dem Sturm und Drang und seinem Genieverständnis und Naturbegriff wandelt sich gegen Ende des 18. Jahrhunderts die überwiegend abwertende Beurteilung von Paracelsus als Mensch. Für Hensler (1783), der sich für eine Verbindung von Medizingeschichte und Philosophieentwicklung einsetzt, ist Paracelsus «ein trefliches Genie ... keines von heuriger Aernte, kränkelnd und wintzig -, ein wahres Genie, umfassend, weit- und schnellsichtig, vest, voll Kraft und Feuer» ${ }^{44}$. Auch Metzger (1792) äussert sich in diesem Sinne beeindruckt, man müsse zugeben, «dass er ein vorzügliches Genie war, fest und weitumfassend; dass er sich dem Strom der Vorurtheile mit Macht entgegen stemmte; dass er den noch immer angebeteten Götzen Galen mit umstürtzen half» ${ }^{45}$; zugleich habe sich bei ihm das Genie nicht nur zum Guten ausgewirkt: «Den grösten Schaden aber stiftete der Schwärmer Paracelsus, welcher die Träumereien der Theosophie auf Alchymie, Naturlehre und Arzneygelahrsamkeit anwendete» ${ }^{46}$.

Wiederholt wird im aufklärerischen Geist des 18. Jahrhunderts für ein gerechteres Verständnis von Persönlichkeit wie Werk des Paracelsus verlangt, die Verhältnisse zu berücksichtigen, in denen Paracelsus lebte. Allgemein müssen nach Barchusen Zeit und Personen beachtet werden, mit denen Paracelsus im Kontakt gestanden habe: «Consideranti enim aevum, in quo vitam vixit, et homines, quibuscum Paracelsus plurimum, ut perhibent, versatus est, minime mirum videbitur, eum adeo multa vaniloqia operibus suis inseruisse» ${ }^{47}$. In Übereinstimmung mit seinem aufklärerisch-pragmatischen Verständnis von Geschichte will auch Sprengel Paracelsus «nur in Rücksicht auf sein Zeitalter und auf den Geist desselben» ${ }^{48}$ beurteilt wissen.

Dass Personen und Werk auseinanderzuhalten sind, wird im 18. Jahrhundert mehrfach betont. Wichtiger als das Leben ist nach Reimmann die Lehre: «Die Historia der Aerzte muss die Historia der Artzney-Kunst nicht 
unterdrücken» ${ }^{49}$. Allen herabziehenden Anekdoten wird von ihm entgegengehalten: «Indeßen wie dem allen, so ist er zu einer ungemeinen Wissenschafft und Erkäntniß in der Artzneykunst gedien» ${ }^{50}$. Im Werk sind wiederum verschiedene Bereiche oder Fachrichtungen ebenso für sich zu betrachten wie in der Wirkungsgeschichte.

Ein Thema für sich ist der paracelsische Sprachgebrauch, der im allgemeinen, aber ebenfalls wieder nicht durchgängig, auf Unverständnis und Ablehnung der Naturforscher und Mediziner des 18. Jahrhunderts stösst. Nach Barchusen hat Paracelsus die Sprache missbraucht und ohne besondere Notwendigkeit neue Ausdrücke geschaffen : «Mira plerumque Paracelso est vocabulorum abusio, quid mirum? erat enim licentiosus sine urgente necessitate novorum vocabulorum fictor» ${ }^{51}$. Albinus (1711) hält Paracelsus für den Urheber einer neuen Medizin («planè novae medicinae auctor»), der neue Bezeichnungen in die neue Wissenschaft eingeführt habe, mit denen er bislang unbekannte Dinge erklärte («quibus res hactenus ignorates explicabat» ${ }^{52}$ ). Nach Le Clerc hat Paracelsus einerseits bekannten Worten einen neuen Sinn gegeben und andererseits habe es ihm gefallen, «à forger des noms barbares qui ne sont tirez d'aucunes langues connues, et dont personne ne sauroit montrer l'origine, tels que sont ceux de Paramirum, de Paragranum» ${ }^{53}$. Die endgültige Beurteilung der oft barbarischen Sprache von Paracelsus möchte Astruc (1740) denen überlassen, «qui scavent l'Allemand ou le Suisse, et qui font cas des niaiseries de Paracelse» ${ }^{54}$. Nach Zimmermann - in wörtlicher Übernahme aus Le Clerc ohne entsprechende Kennzeichnung - hat Paracelsus bewusst den Geist der Sprache verletzt: «Die Sprache ist sonst dem Menschen gegeben sich andern Menschen verständlich zu machen; Paracelsus redte und schrieb um von niemand verstanden und von allen bewundert zu seyn ${ }^{55}$.

Stolle (1731) ist sich sicher: «Hätte er sauber latein geschrieben und geredet, seine Dinge wohl zusammen zu hengen und deutlich vorzustellen wissen, würde er noch mehr Eingang gefunden haben» ${ }^{56}$. Hufeland (1797), bereits mit Blick auf die beginnende Phase der Naturphilosophie, hebt ironisch einen Vorteil der paracelsischen Sprache hervor: «Aber er hatte die Gabe, seinen Unsinn in einer so dunkeln und mystischen Sprache vorzutragen, dass man die tiefsten Geheimnisse darinne ahndete, und noch hie und da darinnen sucht, und dass es wenigstens ganz unmöglich war, ihn zu widerlegen» ${ }^{57}$.

Vor allem die Chemie wird als Dokument der wissenschaftshistorischen Bedeutung von Paracelsus angeführt. Die Rolle von Paracelsus in der 
Chemie will Venel mit der des Aristoteles in der Philosophie vergleichen können; wie immer auch seine chemischen Beiträge insgesamt zu beurteilen seien, die Herstellung von Heilmitteln sei wesentlich ihm zu verdanken ${ }^{58}$. Verdienste möchte Carbonarius Paracelsus ebenfalls besonders in der Chemie zugestehen: «Die Scheidekunst hat durch ihn viel - unendlich viel gewonnen» ${ }^{59}$. Anerkennung bringt auch Gmelin in seiner «Geschichte der Chemie> (1797) Paracelsus entgegen: «ein Mann von ausserordentlichen Naturgaben, aber auch ein Schwärmer der ersten Grösse» ${ }^{60}$.

Von der Chemie wiederum soll der fundamentale Einfluss von Paracelsus auf die Medizin abhängen. Für Albinus hat Paracelsus mit der Einführung chemischer Prinzipien und Medikamente die Medizin tiefgreifend umgestaltet («audacissimo conatu, chemica principia et medicamenta in eam introducendo, illam e fundamentis concutiebat ${ }^{61}$ ). Black erinnert an den Ruf von Paracelsus, «der berühmte Chemiker und Empyriker des fünfzehnten Jahrhunderts» und Urheber «wunderbarer Kuren» ${ }^{62}$ gewesen zu sein; durch sein Beispiel und Ansehen habe er es dahin gebracht, «dass man Spießglas und Quecksilber auch innerlich brauchte und gab» ${ }^{63}$. Bergman zählt dagegen Paracelsus und van Helmont zu den «most extravagant» Anhängern der Lehre von der Universalmedizin; mit ihren heftigen Arzneien («violent medicines») hätten sie bei anderen wie sich selbst aber keinen Erfolg gehabt und damit die Eitelkeit ihrer Lehre und Praxis («vanity of their doctrine and practice» ${ }^{64}$ ) bewiesen. Nach Wiegleb (1777) verdient Paracelsus ungeachtet seines Lebenswandels Dank, weil er «vieles zu der nachfolgenden Reform der Wissenschaften beygetragen ${ }^{65}$ habe.

Clifton (1732) erklärt - auf der Grundlage von Le Clerc und Freind und mit dem Ziel, zur Verbesserung der Medizin beizutragen - Paracelsus dagegen gerade wegen seiner Chemieposition zu einer Behinderung für den neuen Aufschwung der Medizin: "Chemistry, that ist capable of being made so serviceable to physick, was shamefully abus'd by a set of ignorant enthusiastical men with Paracelsus at the head of 'em» ${ }^{66}$. Von dem Portugiesen Barea (1759) wird wie von vielen anderen Naturforschern und Medizinern der Zeit Paracelsus als Kritiker der Humoralpathologie und Begründer der modernen Chemieatrie gewürdigt : «Durò esta paz, hasta que Theofrasto Paracelso introduxo la Chimica en el mismo centro de la Medicina». Vor allem hätte van Helmont zum Erfolg dieser neuen paracelsischen Richtung beigetragen: «Helmoncio solo bastò para sotener el nuevo systèma de Paracelso» ${ }^{67}$.

Das Medizinkonzept von Paracelsus wird im 18. Jahrhundert wiederholt, aber weniger umfassend oder nicht in seiner naturphilosophisch-anthropolo- 
gischen Ganzheitlichkeit, wie das in der Epoche der Romantik und des Idealismus geschieht, dargestellt.

Reimmann referiert den paracelsischen Aufbau der Medizin mit den vier Säulen, geht ausdrücklich auch auf die Tugend des Arztes als der vierten Säule ein, hebt die Verdienste von Paracelsus hervor, als erster die deutsche Sprache in den philosophischen Wissenschaften benutzt zu haben und ein Gegner der Humoralpathologie gewesen zu sein. Von Barchusen wird zwar der ontologische Ansatz von Paracelsus abgelehnt («De Ente, cui neglecto scholarum more, inusitatam significationem tribuit, singularem opinionem habet» ${ }^{68}$ ), zugleich aber betont, dass trotz zahlreicher Einwände im einzelnen seine Lehre vollständig nicht verworfen werden dürfe.

Paracelsus hat nach Le Clerc das Ziel verfolgt, «d'établir une Medecine toute nouvelle sur les ruines de l'ancienne, qu'il s'efforca de renverser de fond en comble» ${ }^{69}$. Bei allem Unsinn und Aberglauben müsse man Paracelsus Verdienste um den Fortschritt der Medizin zugestehen: «parmi tant de mauvaises choses dont ils sont remplis, il s'en trouve quelques unes de bonnes, et qui ont servi à l'avancement de la Médecine» ${ }^{70}$. Eloy hält sich eng an Le Clerc und diesen Interpretationsansatz, wenn er schreibt: «Entre les absurdités dont ses Ouvrages sont remplis, on trouve quelques bonnes choses qui ont servi aux progrès de la Médecine» ${ }^{71}$, wie z. B. seine Kritik an der Humoralpathologie, seine Einführung der chemischen Grundprinzipien (sel, soufre, mercure) in die Medizin. Bacons Urteil sei «juste, quoique sévére» ${ }^{72}$ gewesen. Die Schüler des Paracelsus hätten seine Dogmen allerdings mit der Religion verbunden, «dont ils emprunterent le despotisme, la pompe et les mistères» ${ }^{73}$.

Von Kestner werden in seinem Bericht wesentliche Einzelaspekte mit der allgemeinen Intention verbunden: «Er wolte die gesammte Medicin auf einen bessern Fuß sezen, leitete zuerst die Krankheiten aus dem Tartaro und principiis chemicis her, verwarff die ganze Diaetetic, führte bey der Pharmacie lauter chemische Arzeneymittel ein, und verlachte die so genannten Galenischen Medicamenten, meinte die Würkungen der Simplicium aus ihrer äusserlichen Farbe und Gestalt zu entdecken, hielt von der Chirurgia incisoria und ustoria nichts, sondern machte zuerst von der Chirurgia Sympathetica viel rühmens, und suchte überall die von ihm erfundenen Panaceen nach Art der Charlatans bis in Himmel zu erheben» ${ }^{74}$.

Die Urteile über die Medizin von Paracelsus bleiben in den Beiträgen des 18. Jahrhunderts nicht pauschal, sondern werden im Blick auf einzelne Leistungen konkretisiert. Unzweifelhaft ist nach Le Clerc Paracelsus ein 
grosser Kenner der Materia Medica gewesen. Astruc erwähnt Paracelsus mit einer syphilitischen Krankengeschichte und entsprechenden Krankheitstheorie, die ihn allerdings nicht überzeugt ${ }^{75}$. Paracelsus hat sich nach Eloy gut in der Chirurgie wie allgemein in der medizinischen Praxis ausgekannt: «Disons pourtant à sa gloire, qu'il entendoit très-bien la Chirurgie, et qu'il opéra avec beaucoup de succès; qu'il connoissoit la pratique de la Médicine, aussi-bien qu'aucun de ses contemporains» ${ }^{76}$. Auch nach Gren (1798), der wiederholt in seinen chemisch-pharmazeutischen Schriften Paracelsus erwähnt, gebührt ihm «der Ruhm, dass er uns mit mehrern kräftigen Arzeneymitteln bekannt macht, dass er durch seine Neuerungen mehreres Selbstdenken veranlasste, und Gelegenheit gab, dass der vernünftigere Teil unter den Aerzten eine mehrere Auswahl zu treffen, und den Umfang und die Menge unnützer Mittel mehr einzuschränken anfieng» ${ }^{77}$. Während das Medizinsystem für Scuderi (1794), der grundsätzlich Philosophie aus der Medizin heraushalten möchte, Ergebnis einer «bruta e sregolata fantasia» ${ }^{78}$ sei, könne Paracelsus aber das Verdienst zugesprochen werden, «avere inculcato e posto in voga l'uso di alcuni rimedij attivi, come a dire dell'oppoio, del mercurio, dell'antimonio, dello zolfo, del ferro» ${ }^{79}$. Osterhausen zieht in seiner Schrift «Ueber medicinische Aufklärung〉 (1798) ebenfalls im Blick auf spezifische Arzneimittel einen anerkennenden Schluss: «So viele Vorwürfe diesem Mann auch mit allem Recht gemacht werden können, so ist doch nicht zu läugnen, dass er sich um die Medicin einiges Verdienst erworben hat, wenn ich auch nichts hierher rechnen wollte, als dass er die unwirksamen Syrupe und Abkochungen zu verdrängen und dafür die kräftiger mineralischen Arzneimittel einzuführen suchte» ${ }^{80}$.

Beim Abwägen der positiven und negativen Leistungen wie Auswirkungen werden abweichende Schlussfolgerungen gezogen. Den harten Urteilen von Astruc und Zimmermann möchte Metzger zwar nicht folgen, mit dem theosophischen Unsinn in seinen Schriften habe Paracelsus aber insgesamt «mehr Fluch als Seegen über die Arzney-Wissenschaft gebracht» ${ }^{81}$. Fruchtbare Impulse in verschiedenen Bereichen der Chemie und Medizin erwartet dagegen Hemmann: «es liegen in seinen Werken noch sehr viele hin und wieder zerstreute unentwickelte Keime, welche zu den wichtigsten Untersuchungen und Entdeckungen in der Chymie und Medicin Gelegenheit geben könnten» ${ }^{82}$. Sprengel schliesslich fordert in seinem für die Aufklärung repräsentativen (Versuch einer pragmatischen Geschichte der Arzneykunde〉 (1792-1803) eine «unparteiliche Geschichte dieses paradoxen und merkwürdigen Mannes» ${ }^{83}$ Das Urteil sei über Paracelsus bislang überaus wider- 
sprüchlich ausgefallen : «Wenige Menschen sind durch so viele gute und böse Gerüchte gegangen, und von der einen Seite so ausserordentlich gepriesen, von der andern aber so tief herab gesetzt worden, als eben dieser Vater der Chemiker und Schwärmer unserer Zeiten» ${ }^{84}$.

\section{Perspektiven}

Darstellung und Deutung des Paracelsus in der Epoche der Aufklärung, in der Lexikographie, Philosophie, Naturforschung und Medizin sind vielfältig, differenzieren nach Leben und Persönlichkeit, Werk und Wirkung, sind keineswegs nur von negativen Urteilen bestimmt. Einige Grundzüge und Details konnten in dieser Studie an ausgesuchten Beispielen vorgetragen werden; weitere Aufgaben stehen noch aus, weitere Entdeckungen werden noch zu machen sein. Insbesondere sollte die Rezeption am Werk überprüft werden; wurde Paracelsus angemessen aufgenommen, war das Missverständnis produktiv, über wen fand die Vermittlung statt? Mit diesen Fragen wird der Blick wieder auf die Rezipienten zurückgelenkt, auf die Bedingungen ihrer Aufnahme oder Aktualisierung von Paracelsus.

Im Unterschied zur Aufklärung ist das Bild des Paracelsus in der Naturphilosophie, Naturforschung und Medizin der romantisch-idealistischen Epoche von einer umfassenden Deutung und einer geistigen Verbundenheit geprägt ${ }^{85}$. Metaphysische Orientierung, ganzheitlicher Ansatz von Kosmologie und Anthropologie, biochemische Perspektive, Hochschätzung der Ethik, innere Einheit von Ätiologie, Pathophänomenologie und Therapie bestimmen das Bild von Paracelsus in dieser Zeit. Paracelsus repräsentiert den Übergang vom Mittelalter zur Neuzeit, Person und Leben, keineswegs frei von bedenklichen Zügen, sollen in den Zeitverhältnissen, auf die sie bezogen sind, aber nicht aufgehen, Paracelsus wird in seiner wissenschaftshistorischen Bedeutung und nicht seinem moralischen Verhalten beurteilt.

Die Ablehnung von Idealismus und Romantik lässt es in der Naturforschung und Medizin des positivistischen oder naturwissenschaftlichen 19. Jahrhunderts zu einem neuen Urteil über Paracelsus kommen, das in manchen Zügen an Auffassungen des 18. Jahrhunderts wieder anknüpft. Durchgängig erscheinen aber auch in jener naturphilosophischen Epoche um 1800 naturwissenschaftliche und medizinische Schriften, die sich von metaphysischen Einflüssen freihalten oder diese ausdrücklich verurteilen; umgekehrt werden auch noch nach 1830 naturwissenschaftliche und medizi- 
nische Beiträge sowie vor allem medizinhistorische Werke im Geist der Romatik und des Idealismus publiziert. Ausserhalb Deutschlands dominiert ohnehin um 1800 eine empirisch-positivistische Wissenschaftsauffassung und Historiographie mit einem entsprechenden Paracelsusbild. Diese komplexe Situation soll in einer eigenen Abhandlung (Paracelsus im Urteil der Naturwissenschaften, Medizin und Wissenschaftshistoriographie des 19. Jahrhunderts> dargestellt werden.

Mit der Paracelsus Edition und den Beiträgen von Sudhoff setzt um 1900 eine neue Epoche der Paracelsusforschung ein, auf die auch das gegenwärtige medizin- und wissenschaftshistorische Studium wesentlich bezogen ist. Ein Thema für sich stellt die Beurteilung von Paracelsus in den Naturwissenschaften und der Medizin des 20. Jahrhunderts dar, die zur wissenschaftshistorischen Literatur zunehmend die Verbindung verloren haben. Diese Trennung trifft auch gegenüber Philosophie, Theologie und allgemeine Geschichtswissenschaft zu, die allerdings ihrerseits mit den Naturwissenschaften, der Medizin und ihrer Historiographie kaum noch in einem Kontakt stehen.

Die Interpretationen von Paracelsus aus jenen zurückliegenden Epochen der Aufklärung, der Romantik und des Positivismus verdienen auch heute noch das Interesse - nicht nur der Philologie oder Medizin- und Wissenschaftsgeschichte. Vor allem Romantik und Idealismus versprechen mit ihrer spezifischen Verbindung von Kosmologie, Anthropologie und Transzendenz gegenwärtigen wissenschaftshistorischen wie wissenschaftstheoretischen Analysen mit ihrer positivistisch-spezialisierten Orientierung oder philologischen Zurückhaltung interpretatorische Anregungen.

\section{Anmerkungen und Literaturhinweise}

1 Zur Rezeption im 18. und 19.Jahrhundert vgl.: W. Artelt, Paracelsus im Urteil der Medizinhistorik, in Fortschritte der Medizin 50, 1932, p. 929-933; W. Artelt, Wandel des Paracelsusbildes in der Medizingeschichte, in Nova Acta Paracelsica 8, 1957, p.33-38; L.Braun, Paracelsus und die Philosophiegeschichte, Wien 1965 (= Salzburger Beiträge zur Paracelsusforschung, 5); A.G. Debus, The French Paracelsians, Cambridge 1991; T.Gerecke, Paracelsus im Urteil der vergangenen Jahrhunderte und seine Würdigung in der Neuzeit, med. Diss. Freiburg 1945; P. Dilg u. H. Rudolph, ed., Resultate und Desiderate der Paracelsus-Forschung, Stuttgart 1993 (= Sudhoffs Archiv Beihefte 31); S. Domandl, ed., Paracelsus, Werk und Wirkung, Wien 1975 (= Salzburger Beiträge zur Paracelsusforschung, 13); D. v. Engelhardt, Paracelsus im Urteil der Naturforschung und Medizin der Romantik, in NTM. Internationale Zeitschrift für Geschichte und Ethik der Naturwissenschaften, Technik und Medizin, N. S., 2, 1994, p. 1-20; K. Ganzinger, Das Paracelsus-Bild im 
pharmazeutischen Schrifttum des späten 18. Jahrhunderts, in Österreichische Apotheker Zeitung 30, 1976, p.93-97; K. Goldammer, Paracelsus in der deutschen Romantik. Eine Untersuchung zur Geschichte der Paracelsus-Rezeption und zu geistesgeschichtlichen Hintergründen der Romantik, Wien 1980 (= Salzburger Beiträge zur Paracelsusforschung, 20); R. Herbertz, Paracelsus bei Schopenhauer, in Jahrbuch der Schopenhauer-Gesellschaft 34, 1951-52, p. 64-68; J. M. López Piñero, Eighteenth century medical vitalism. The Paracelsian connection, in W. R. Shea, ed., Revolutions in Science. Their Meaning and Relevance, Canton, Mass., 1988, p.117-132; R.P. Multhauf, Medical Chemistry and 'The Paracelsians), in Bulletin of the History of Medicine 28, 1954, p.101-126; W.Pagel, The smiling spleen. Paracelsianism in Storm and Stress, Basel 1984; J. Strebel, Paracelsus und Schopenhauer, in Praxis 39, 1950, p.331-339; J. Telle, ed., Parerga Paracelsica. Paracelsus in Vergangenheit und Gegenwart, Stuttgart 1992; K.-H. Weimann, Paracelsus in der Weltliteratur (Beiträge zur Wirkungsgeschichte Hohenheims), in Germanisch-Romanische Monatsschrift 42, 1961, p. 241-274; K.-H. Weimann, Paracelsus bei Leibniz, in Die ganze Welt eine Apotheke. Festschrift für Otto Zekert, ed. v. S. Domandl, Wien 1969 (= Salzburger Beiträge zur Paracelsusforschung, 8), p. 221-134; F. Weinhandl, Paracelsusstudien, Wien 1970 (= Salzburger Beiträge zur Parcelsusforschung, 10).

2 A. Teissier, Les Eloges des hommes savans, tirez de l'histoire de M. de Thou avec des additions, Genf 1683, Leyden ${ }^{4} 1715$, Vol. 3, p. 137-147.

3 H. Conring, Introductio in universam artem medicam, Helmstedt $1654,{ }^{2} 1687$, erneut Halle 1726, p. 111; fast gleichlautend bei C. J. Langius, Historia medica, in Opera omnia medica theoretico-practica, Leipzig 1704, p. 26.

4 D. G. Morhof, Polyhistor, Vol. 1, Buch 1, Cap. 10, Lübeck ${ }^{2} 1695$, p. 87.

5 L. di Capoa, Parere, divisato in otto ragionamenti, ne' quali partitamente narrandosi l'origine, e'l progresso della medicina, Neapel 1681, ${ }^{2} 1689,{ }^{3} 1695$, auch Bologna 1714, Zitat ${ }^{2} 1689$, p. 28.

6 E. Chambers, Cyclopaedia, or, an universal dictionary of arts and sciences, Vol.1, London 1728, p. 217.

7 J.H. Zedler, Grosses vollständiges Universal-Lexikon, Vol.26, Halle und Leipzig 1740, Sp. $720-722$.

8 Ibid., Sp. 721.

9 C. G. Jöcher, Allgemeines Gelehrten-Lexikon, Vol.3, Leipzig 1751, p. 1245 f.

10 D. Diderot u. J. le R. d'Alembert, Encyclopédie ou dictionnaire raisonné des sciences, des arts et des métiers, Vol.10, Amsterdam 1765, p. 273.

11 H.Suden (= Erdmann Uhsen), Der gelehrte Criticus, Th. 3, Leipzig 1717, p. 998.

12 Ibid., p. 1011.

13 J.M. Schröckh, Abbildungen und Lebensbeschreibungen berühmter Gelehrten, Leipzig 1764, p. 13.

14 A. Savérien, Histoires des philosophes modernes, Vol. 7, Paris 1769, p.9.

15 Ibid., p. 34 .

16 Ibid., p. 35.

17 S.Domandl, Paracelsus: Stationen deutscher Philosophie. Nikolaus von Kues, Paracelsus, Leibniz, Kant, Goethe, Wien 1990 (= Salzburger Beiträge zur Paracelsusforschung, 27); Weimann, s. Anm. 1), 1969.

18 J. G. Walch, Philosophisches Lexicon, Leipzig 1726, ${ }^{4} 1775$, Sp. 1403. 
19 Ibid., Sp. 203; vgl. a. Sp. 3045.

20 J. Brucker, Historia critica philosphiae, Vol.4, T. 1, Leipzig 1743, p. 663.

21 Ibid., p. 666.

22 J.C. Adelung, Theophrastus Paracelsus, ein Kabbalist und Charlatan, in Geschichte der menschlichen Narrheit, 7. Theil, Leipzig 1789, p.194.

23 Ibid., p. 195.

24 Ibid., p. 289.

25 Ibid., p. 290.

26 C. M. Wieland, Einige Nachrichten von Theophrastus Paracelsus, in Der Teutsche Merkur, 1776, p. 85.

27 I. Kant, Reflexionen zur Anthropologie, handschriftlicher Nachlass, in Gesammelte Schriften, Vol.15, Berlin 1923, p. 8; vgl. a. J.G. von Herder, Aelteste Urkunde des Menschengeschlechts, Vol. 1, 1774, in Sämtliche Werke, Vol. 6, Berlin 1883, Nachdruck Hildesheim 1967, p. 196.

28 J. G. von Herder, Kritische Wälder, 1769, in Sämtliche Werke, Vol.3, Berlin 1878, Nachdruck Hildesheim 1967, p. 261.

29 A. von Haller, Bibliotheca chirurgica, Vol.1, Bern 1774, p. 183.

30 C. G. v. Murr, Litterargeschichte des Theophrastus Paracelsus, in Neues Journal zur Litteratur und Kunstgeschichte 2, 1799, p. 180.

31 J. C. G. Ackermann, Institutiones historiae medicinae, Nürnberg 1792, p. 404.

32 J.F. Reimmann, Versuch einer Einleitung in die Historiam literariam, Th.3, 4. Hauptst., Halle 1713, p. 556.

33 D. Le Clerc, Histoire de la médecine, Genf 1696, Amsterdam 1702, ${ }^{2} 1723$, p. 802 f.

34 H. Boerhaave, Elementa chemiae, Vol. 1, Leipzig 1732, p. 23.

35 N. F. J. Eloy, Paracelse, in Dictionnaire historique de la médecine, Vol.2, Liège 1755, p. 247.

36 G.F. Venel, Chymie, in Encyclopédie ou dictionnaire raisonné des sciences, des arts et des métiers, Vol.3, Paris 1753, p. 431.

37 J. G. Zimmermann, Von der Erfahrung in der Arzneykunst, Th. 1, Zürich 1763, p. 121.

38 Carbonarius (?C. G. v. Murr), Des grossen Theophrastus Paracelsus, Leben, Meynungen, Verdienste, Schicksale und Schriftstellerarbeiten, in Beytrag zur Geschichte der höheren Chymie, Leipzig 1785, p. 163.

39 Ibid., p. 174.

40 Ibid., p. 178.

41 Ibid., p. 183.

42 J. F. Blumenbach, Introductio in historiam medicinae litterariam, Göttingen 1786, p. 139.

43 Ibid., p. 140.

44 P.G. Hensler, Geschichte der Lustseuche, die zu Ende des XV.Jahrhunderts in Europa ausbrach, Altona 1783, p. $128 \mathrm{f}$.

45 J. D. Metzger, Skizze einer pragmatischen Literärgeschichte der Medicin, Königsberg 1792, p. 195.

46 Ibid., p. 173.

47 J. C. Barchusen, De medicinae origine et progressu dissertationes, Utrecht 1713, p. 397.

48 K.Sprengel, Versuch einer pragmatischen Geschichte der Arzneikunde, Vol.3, Halle 1794, p. 335 .

49 Reimmann, s. Anm.32), p. 564. 
$50 \quad$ Ibid., p. 554 .

51 Barchusen, s. Anm. 47), p. 393.

52 B. Albinus, Oratio de incrementis et statu artis medicae seculi decimi septimi, Leyden 1711, p. 33 .

53 Le Clerc, s. Anm. 33), p. 804.

54 J. Astruc, Traité des maladies vénériens, Vol. 3, Paris $1740,{ }^{2} 1743,{ }^{3} 1743$, p. 265.

55 Zimmermann, s. Anm.37), p.121.

56 G.Stolle, Anleitung zur Historie der medicinischen Gelahrtheit, Jena 1731, p. 171.

57 Hufeland, Jena 1797, p.16.

58 Venel, s.Anm. 36), p. 431.

59 Carbonarius, s. Anm. 38), p. 185.

60 J.F. Gmelin, Geschichte der Chemie seit dem Wiederaufleben der Wissenschaften bis an das Ende des achtzehenden Jahrhunderts, Vol.1, Göttingen 1797; Nachdruck Hildesheim 1965, p. 195.

61 Albinus, s. Anm. 52), p. 33.

62 W. Black, An historical sketch of medicine and surgery from their origine to the present time, London 1782, dt. Lemgo 1789, p. 232.

63 Ibid., p. 309.

64 T. Bergman, The history of chemistry during the obscure or middle age, a. d. Lat. (1782), in Physical and chemical works, Vol. 3, London 1791, p. 145.

65 J. C. Wiegleb, Historisch-kritische Untersuchung der Alchemie, Weimar 1777, p. 234; vgl. a. Wiegleb, Handbuch der allgemeinen Chemie, Berlin 1781; sowie Fussnoten zu Bergman, Anm. 64), dt. Berlin 1792.

66 F. Clifton, The state of physick, ancient and modern, briefly consider'd with a plan for the improvement of it, London 1732, p. 117.

67 M. F. Barea, Historia interior de la medicina, Malaga 1759, p. 27.

68 Barchusen, s. Anm.47), 1713, p. 380.

69 Le Clerc, s. Anm. 33), ${ }^{2} 1723$, p.792.

70 Ibid., p. 818.

71 Eloy, s. Anm.35), Vol.2, 1755, p. 247.

72 Ibid., p. 248.

73 Ibid., p. 245.

74 Kestner, Medicinisches Gelehrten-Lexikon, Jena 1740, Nachdruck Hildesheim 1971, p. 617; in seinem 〈Kurzen Begriff der Historie der Medicinischen Gelahrtheit) (1748, p. 215) wird von Kestner nur die Ablehnung der griechischen und arabischen Ärzte durch Paracelsus und seine chemische Grundlegung der Medizin erwähnt.

75 Astruc, s. Anm. 54), Vol. 1, ${ }^{3} 1743$, p. 244, 246, 248, 331, 334.

76 Eloy, s. Anm. 35), p.246f.

77 F. A. C. Gren, System der Pharmacologie, Vol.1, Halle 1798, p. 50.

78 R.Scuderi, Introduzione alla storia della medicina antica e moderna, Neapel 1794, Padua ${ }^{2} 1824$, p. 44.

79 Ibid., p. 45.

80 J. K. Osterhausen, Über medicinische Aufklärung, Zürich 1798, p. 332.

81 Metzger, s. Anm.45), p. 195. 
82 J.A. Hemmann, Beitrag zur Geschichte der Magnetkur, aus den Werken des Theophrastus Paracelsus, in Medicinisch-chirurgische Aufsätze historisch-practischen Inhalts, Berlin 1778, p. 22 .

83 Sprengel, s. Anm.48), p. 337.

84 Ibid., p. 336.

85 v. Engelhardt, s. Anm. 1).

Prof. Dr. D. von Engelhardt

Institut für Medizin- und Wissenschaftsgeschichte Königstrasse 42

D-23552 Lübeck 